\title{
An Architecture for Blockchain over Edge-enabled IoT for Smart Circular Cities
}

\author{
Amalia Damianou \\ Dep. of Computing \& Informatics \\ Bournemouth University \\ Poole, UK \\ adamianou@ bournemouth.ac.uk
}

\author{
Constantinos Marios Angelopoulos \\ Dep. of Computing \& Informatics \\ Bournemouth University \\ Poole, UK \\ mangelopoulos@bournemouth.ac.uk
}

\author{
Vasilis Katos \\ Dep. of Computing \& Informatics \\ Bournemouth University \\ Poole, UK \\ vkatos@bournemouth.ac.uk
}

\begin{abstract}
Circular Economy is a novel economic model, where every 'asset' is not wasted but reused and upscaled. The Internet of Things-IoT paradigm can underpin the transition to a Circular Economy by enabling fine-grained and continuous asset tracking. However, there are issues related to security and privacy of IoT devices that generate and handle sensitive and personal data. The use of Blockchain technology provides an answer to this issue, however, its application raises issues related to the highly-constrained nature of these networks. In this paper, Edge Computing is presented as a solution to this issue, providing a way in which Blockchain and Edge Computing can be used together to address the constrained nature of IoT. Furthermore, we present the challenges that this combination poses and the opportunities that it brings. We propose an architecture that decreases the IoT devices requirements for memory capacity and increases the overall performance. We also discuss the architecture design and the challenges that it has, comparing it to the traditional Blockchain architecture as well as an Edge Computing architecture for Mobile Blockchain. The paper closes with a discussion and future extensions of our work are presented, as well.
\end{abstract}

Index Terms-Circular Economy, Internet of Things (IoT), Blockchain Technology, Edge Computing

\section{INTRODUCTION}

As the population of cities becomes bigger and bigger, the number of challenges that these cities should face increases. These challenges are related to transport means, urban population, healthcare, citizens safety and criminality, energy, the industrial sector, economy, financial transactions, etc. In order for these challenges to be addressed, all parts and members could play an active role in their own city towards improving the urban conditions. In real cases, this concept could be enhanced using a novel economic model; Circular Economy. Circular Economy model minims waste by reusing most of resources and provisions the use of emerging ICT technologies to achieve its main goals. The paradigm of the Internet of Things provisions networks of physical devices, like sensors, cameras, smart devices, like smartphones, vehicles, etc., which obtain unique identifiers and can be connected to the Internet and exchange data between each other seamlessly. In Circular
Economy, IoT can contribute to data collection, machine to machine communication and exchange of data, asset tracking, energy management and in many other cases. However, IoT networks face several security and privacy vulnerabilities that can be exploited to compromise IoT devices. Furthermore, the performance of some services of IoT devices could be enhanced and improved. For example, the availability and usage of an asset, which is part of an IoT network can be regulated via smart contracts. For this reason, Blockchain can be used. Blockchain is a new, well-known technology that first appeared as a fundamental part of Bitcoin cryptocurrency [8]. It has emerged as a promising technology that has been adopted not only for digital currencies purposes but in many other applications as well, like in healthcare and transportation sectors. However, the adoption of this new technology is not a panacea and may raise significant issues. In the case of Blockchain over IoT, significant challenges are posed from the highly-constrained nature of IoT devices in terms of available computational and communication resources, available energy resources, etc. For instance, in Blockchain applications, each node of the network maintains a copy of the ledger, which may grow significantly in size (in some cases even in the order of several GBs [21]). Furthermore, Blockchain applications are contradicted the IoT paradigm. In order to address these issues, Edge Computing can be employed.

Our contribution. We present a novel and innovative hybrid blockchain-based architecture for IoT networks, where the ledger is stored on the Edge Computing nodes and not on the nodes of the Blockchain network. We discuss the technologies that will be employed and the main idea behind this architecture, which is that the devices of the network run a Blockchain application and perform Proof of Work computations, however, the ledger is stored on Edge Computing rather than on the devices. The architecture is critically evaluated against other existing Blockchain architectures and we discuss our findings in the broader context of emerging ICTs and their implications. 


\section{PRELIMINARIES}

\section{A. Circular Economy}

The term Circular Economy is used to describe this economic system, where everything in an environment, like in a smart city, has value and nothing is wasted. "Everything" includes materials, devices, services, and generally resources, and the main goal of Circular Economy is to maintain their utility and use them as long as possible without producing any waste [1]. The main idea behind Circular Economy is to design products in a smart way so as to be used during their whole life cycle, be re-used and re-paired to extend this life cycle and remanufactured to create new products [1]. The concept of Circular Economy was first introduced in the late 1970s and evolved over the years until now from the linear "takemake-dispose" model to the "make, use, remake" model. IoT can help transition to a Circular Economy as it can provide useful information on assets, their location, the condition, and the performance during their whole life cycle, in real time and over time. IoT is already used to address many challenges that Circular Economy faces, mostly regarding the efficient use of resources [2]. The combination of Circular Economy and IoT can provide a fertile ground for the innovative applications of the first, such as the requirements regarding maximising the utility of assets in a more efficient way, creating clusters of "intelligent assets", and so on.

\section{B. Internet of Things (IoT)}

Internet of Things provisions networks of physical devices, like smartphones, vehicles, homes, home appliances, etc., that hold a unique identifier and can transfer data without requiring human intervention. The number of connected IoT devices is ever increasing and is projected to grow to 26 billion by 2020 , which is 30 times the estimated number of devices deployed in 2009 and it is far more than the 7.3 billion smartphones, tablets, and PCs that are expected to be in use by 2020 [1] [3]. Currently, most IoT solutions rely on centralised server-client systems that offer cloud servers solutions connected through the Internet. Even though this approach works properly, there is a need for improvement. As a side effect of its popularity, the number of vulnerabilities and attacks taking advantage of the complexity and the heterogeneity of IoT networks is increasing. This fact raises the need for protecting these networks by applying sophisticated cyber security methods, such as Blockchain. Blockchain allows to execute transactions, track, and apply strong cryptographic techniques to protect the records that are stored on a distributed ledger. For this reason, applying Blockchain over IoT could be a solution forwards mitigating corresponding security issues.

\section{Blockchain Technology}

Blockchain is a distributed ledger that keeps records of transactions and/or data that take part in a network. This ledger is digital and decentralised, which means that it does not rely on any central authority which manages the records of it. This is achieved because the network is peer-to-peer. Everyone is able to join the network, have access to the data, verify transactions and communicate with the other members of it. Because of the decentralised and distributed nature of Blockchain, the records of the ledger can be maintained across different systems, devices, and locations inside the network [10]. The data and generally the records of the Blockchain are secured by applying strong cryptographic techniques over the ledger. Some of these techniques include digital hashes, asymmetric cryptography, and digital signatures [8]. Blockchain is based on the transaction validation process through a distributed network to create and maintain a verified and secure list of information, the ledger. A fundamental process of Blockchain is mining. The mining process starts when miners wish to verify transactions. In order for this process to be achieved, consensus mechanisms must be used. Every block of the chain has a header which includes a hash value as well, apart from the other information. Even though Blockchain can be used over IoT networks in order to provide efficient security and privacy levels, there are still some barriers, as it needs strong computational capabilities and energy consumption.

\section{Edge Computing}

Edge Computing is a new, cloud-based technology that provides an IT services environment at the edge of the network. It uses the Radio Access Network (RAN) and it is closer to mobile users than cloud services or databases [9][6]. The main goal of Edge Computing is to enhance the performance of the network reducing its latency, increasing the efficiency of the network and providing a better experience to its users by being closer to them. The main characteristics of Edge Computing include low latency, proximity, high bandwidth and real-time insight into radio network information and location awareness, as data is collected and processed closer to customers [6]. Implemented applications on Edge Computing require high bandwidth and low latency environments. For this reason, distributed data centres service providers are located at the Edge Computing. Users can have access to Edge Computing in multiple ways, like using wireline. Edge Computing is the evolution of mobile base stations and one of the fundamental technologies behind 5G networks, apart from Network Functions Virtualisation (NFV) and Software-Defined Networking (SDN). It contributes to the demanding requirements regarding throughput, latency, scalability, and automation of 5G, and it helps to improve the mobile broadband network into a programmable world [6].

\section{RELATED WORK}

The growth of big cities population around the world is a fact that comes with many challenges. It is estimated that big cities consume $75 \%$ of natural resources globally and produce $50 \%$ of global waste [17]. In these cases, the model of Circular Economy can help with the reduction of natural resources consumption and waste management. Connected devices can help enhance this scenario collecting and managing data from city sectors, like energy, healthcare, transportation, financial systems, etc. The collection of this data can reveal many useful 
details about the way these sectors work and the needs of each one. In this way, local authorities around the world can use these details in order to ameliorate their operation and provide the levels of resources that each one needs. Circular Economy applications can designate the value of intelligent assets that can contribute to the development of smart cities. In this way, assets maintain their value, providing their services all through their life cycle [17]. As it has been mentioned above, the transition to Circular Economy can be facilitated with the use of IoT network devices. In this case, the connected devices could be devices that are part of this kind of networks, the performance of which could be improved with the application of blockchain.

In many cases of Blockchain applications, there are some barriers regarding the mining process, and the storage of data in these environments, mainly related to processing power and time, energy consumption and other resources, which are limited in IoT and mobile devices. In [4][5], authors describe two new architectures that are based on blockchain in combination with cloud services, as the memory capacity of the IoT devices is limited and the demands of Blockchain regarding data/transactions storage are high. They use cloud services as a storage solution that keeps records of all the transactions inside the network, something that IoT devices are not able to do because of their memory capacity limitations. The only disadvantage here is that in real time scenarios, this solution and architecture may not be efficient enough, as there is need for data in real-time. Furthermore, the response of cloud services to the requests of a smart vehicle, for example, may take more than $5 \mathrm{~ms}$, which means that this process consumes valuable time from the end-user, who need their data in real time and contrasts with the $5 \mathrm{G}$ requirements. For these reasons, we believe that Edge Computing could be a better solution to the issue of memory capacity.

Edge Computing is a new technology and a key enabler for $5 \mathrm{G}$ networks. Edge Computing technologies provide cloudcomputing capabilities and an IT service environment at the edge of the network, closer to end-users than traditional cloud architectures. The main features of this paradigm are low latency, high bandwidth and real-time access to radio network information [14]. IoT networks are the key enabler of many sectors' evolution. One of these is the automotive sector, where many technologies are applied as there is a need for digitalisation of services. As autonomous cars have already emerged, many companies around the world have employed technologies that can increase their performance and transfuse other, more improved features. These technologies are not applied only over smart vehicles but over the smart transportation sector generally. For instance, a traffic alert service has been demonstrated in Germany at Digital A9 Motorway test bed, where a live long term evolution network had been deployed in order to transfer alert messages between vehicles. This experiment was enabled by an Edge Computing solution and the target was the reduction of the latency of sending the messages between the vehicles [14]. Furthermore, in [19], authors present concepts of Edge Computing systems, like
Cloudlet, Fog Computing, and Multi-access Edge Computing, the advantages, and limitations of these concepts and the reason why the improvement of Edge Computing concepts is essential. Furthermore, they propose a novel Edge Computing architecture, the Home Edge Computing that includes three layers, the Home Server, the Edge Computing and the central Cloud. The term "Home" that they use does not refer to the users' homes but to places where users can connect to the Internet. These places may be companies, hospital, malls, etc. The main goal behind this architecture is the reduction of latency that traditional Edge Computing schemes have by employing storage and data processing devices closer to endusers. Furthermore, some of the benefits of this architecture include reduction of the workload on the Edge Computing Server and hierarchical resource allocation, as the request users' flows pass first from Home Servers, and will be transferred to Edge Computing or Central Cloud if it cannot process them. In this way, Edge Computing has fewer spots and task to process. Comparing this new architecture to other existing Edge Computing concepts, authors provide arguments and experimental outcomes that enhance their claims regarding the performance of their architecture.

\section{Model And ARChitecture}

\section{A. Edge Computing for Blockchain-enabled IoT networks in a smart circular city}

As already mentioned, demands of Blockchain on memory capacity and energy consumption are high and IoT devices are not able to perform the computational processes that Blockchain requires. For this reason, Edge Computing is employed as a storage service of Blockchain ledger that is deployed close to the network. The main idea is the need for memory capacity that Blockchain has and cannot be supported by smart, mobile devices. In this case, IoT devices should use their resources to perform mining, like CPU, time and energy. The devices that will be used in our architecture, will be class $\mathrm{C}$, which are IoT devices with significant communication capabilities and processing power with direct access to the Internet. These mobile devices are part of an IoT network and may be single board computers, sensors, cameras, smart devices, like smart traffic lights, smart vehicles, smartphones, etc. These devices are nodes of the Blockchain network and act as miners. However, due to the limited memory capacity that they have, there is a need for alternative solutions regarding the storage of data and generally the storage of the ledger. Furthermore, to prevent privacy issues related to the location where data is stored, and their availability, we support the use of Edge Computing as a solution. To demonstrate the applicability and effectiveness of Edge Computing in a Blockchain environment, we propose an architecture that combines the two technologies and gives fertile ground for the improvement of both, as the Edge Computing has already many promising applications and the Blockchain is promising as well in spite of many existing barriers. The new architecture that we propose is presented at fig. 1. To start with, the devices will be employed to collect, exchange and store 


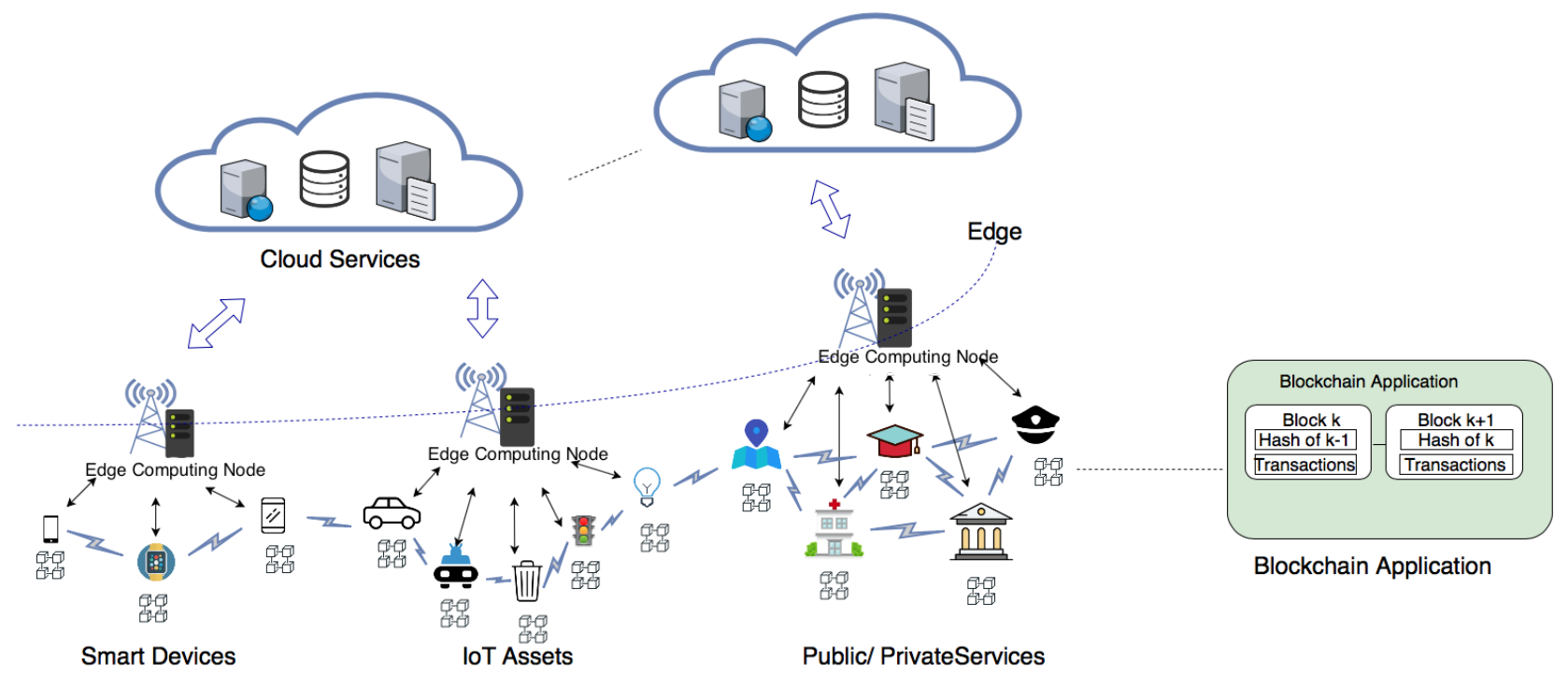

Fig. 1. Edge Computing nodes as storage enablers of Blockchain ledger for IoT networks in a smart circular city

useful data inside a smart, circular city environment running a Blockchain application. All these processes will be conducted in the form of transactions that are relevant to the data that these devices exchange, the communication between them, the storage of data collected, provided services, their interaction with other environments, like with physical and other digitals environments, etc. All the operations will be verified through the mining process of Blockchain. The data that will be included in these transactions, may be location data of mobile devices, (like vehicles, phones, etc.), data regarding availability and condition of these devices, sensors' data that give useful information for interactions with end-users, data regarding weather and climate, etc. As the amount of data and the need for communication become stronger and stronger, devices must produce and verify many transactions in a small period of time. The main problem here is that as many transactions are generated, the number of Blockchain blocks that are generated is also increased proportionally. This has as a consequence the growth of the ledger size that these devices should store. However, these IoT devices have limited memory capacity. The alternatives solution that we introduce is the Edge Computing, which provides IT services environments at the edge of networks. Edge Computing can be used as a ledger storage solution, which means that the transactions will be verified and the generation of new blocks will be conducted in the same way as in traditional Blockchain environments, however, the records of the performance, the verified transactions, the details of each node and the communication between them in the network will not be stored locally by the devices of the network but by the Edge Computing nodes that will be places inside a smart circular city environment. In this case, the nodes on Blockchain network, which are the IoT devices, will act properly as Blockchain nodes, however, they will not store the ledger at their local memory, and they will send information for every stage of each process in the network to Edge Computing nodes. We assume that Edge Computing nodes that we employed are trusted and reliable from the beginning and they will not be managed by any central authority, in order to comply with the basic regulations of Blockchain technology. This means that each IoT device will be able to have access to the ledger that will be kept updated when every new block is generated, however, this ledger will not be stored locally, but, at the Edge Computing nodes. This fact will increase the performance of mobile devices, as the need for more and more memory consumption will be mitigated. The communication between IoT devices and Edge Computing nodes will be performed via LPWAN (low-power wide-area networks), such as LoRa and SigFox, to achieve low levels of latency. Each device maintains a communication channel with Edge Computing nodes and has access to the ledger and every stored data on it. In this way, access to the ledger is faster, with low latency, as each communication channel will serve only one connection, through which each node will be served and will have access to the ledger.

By keeping stored data on Edge Computing nodes, the extraction of it regarding the needs of smart circular cities concepts can become easier, as, in this way, data related to assets, like their condition and their availability can be used in order for the circularity to be achieved. Furthermore, data related to sectors of smart cities, like energy, healthcare, transportation, etc. can be extracted from the stored data on Edge Computing nodes as well, in order to help them to be improved and the resources usage mitigation.

\section{B. Mining process}

The mining process will be conducted by IoT devices, as in traditional Blockchain networks. The difference is that the 
ledger will not be stored on these devices but on the local Edge Computing node. This means that devices that act as miners and need to validate new transactions in the network, is necessary to compute the Proof of Work - PoW value and come to consensus with the other nodes. As the nodes of the network exchange and collect data in a transaction form, in order for a miner to generate a new block, he must first compute the Proof of Work value. However, the mining process includes the hash of the previous mined block in order to achieve the connectivity between the blocks. For this reason, miners should have access to the hash value of the last mined block in order to compute the hash of the new one. As the ledger is stored on the Edge Computing nodes, miners can access this block from there. Devices, which are the nodes of the Blockchain network, will have access to the stored ledger upon request. In the same way, using each communication channel, Edge Computing nodes will be able to return the hash value of the last added block to each of the requesting nodes, whenever they are ready to mine a new block. In this way, all the nodes of the Blockchain network, can sustain their operating role, increasing their performance, as they will dispose their memory capacity without worrying about the growth of the size of the ledger and the availability of the last generated block hash value to mine the new block, verify new transactions and receive the rewards. The new block will be mined by one of the miners of the network and it will be stored on Edge Computing nodes. The generated block will be transferred to the Edge Computing nodes via an LPWAN, and stored there with the other previous mined blocks, keeping the chain updated. After the mining process, the Blockchain node, who first computed the most appropriate Proof of Work value is the miner of the new block. The miner of each block transfers it to the Edge Computing nodes through its LPWAN communication channel and stores it there. In this way, miners keep the Blockchain ledger updated.

\section{COMPARISON TO OTHER BLOCKCHAIN ARCHITECTURES}

The aim of introducing the use of Edge Computing is to improve performance and enable IoT devices to make use of Blockchain. Furthermore, using Blockchain technology, we would like to enhance the security and privacy of IoT networks, as there are many vulnerabilities, which could have a great impact on the performance of these networks, in cases of exploiting them. However, at this point, we would like to identify the advantages and disadvantages of our prototype comparing it with the nominal architecture of Blockchain and the Edge Computing for Mobile Blockchain architecture, which is presented in [13].

\section{A. Nominal Blockchain Architecture}

The term Blockchain is used to describe a sequence of blocks, groups of completed transactions lists, which are kept by the system as records and it is not possible to be changed. All these blocks are stored by all the participants of the network, the nodes, and create a chain of blocks,

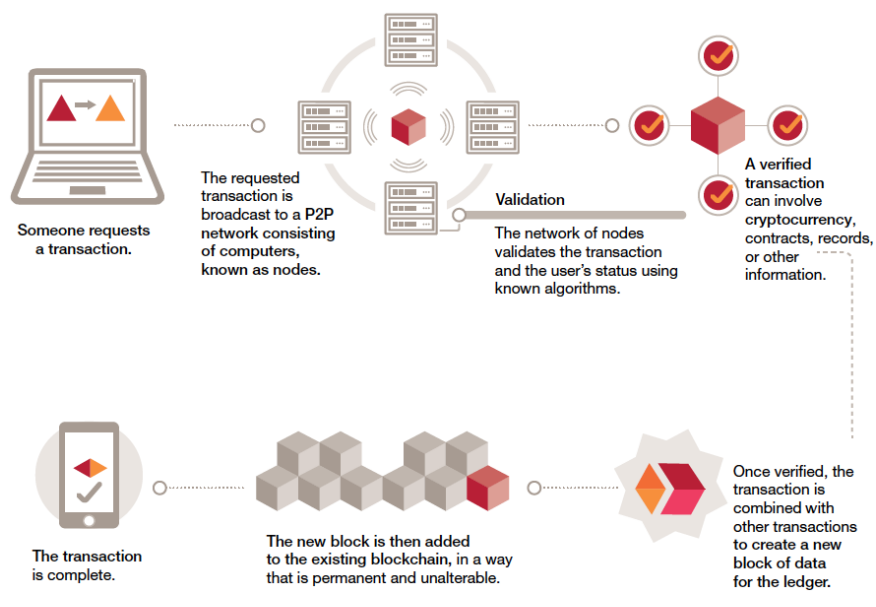

Fig. 2. How Blockchain Works [15]

which is referred to as ledger. Each block constitutes of the block header and the main part of it, which includes a transaction counter and transactions. The header of a new block contains the block header of the previous block, as well. In this way, the sequence of the chain is achieved. Each user of the Blockchain obtains at least one pair of a public and a private key that is used in order to sign and verify the transactions that nodes exchange. The sender signs each transaction using his private key and the receiver can verify this transaction by validating the value with the public key of the sender. In this way, the receiver can check if the data has been tampered. Blockchain is categorised into three types, regarding the access mode to the ledger. These types are the public, private and consortium blockchain. As it has been mentioned above, blockchain is a decentralised system which does not need a central authority to manage it. For this reason, Blockchain uses decentralised consensus mechanisms. In this way, the distributed ledger can guarantee the reliability and the consistency of the data and/or transactions among which are stored on it. There are many consensus mechanisms. The most well-known consensus mechanisms are the Proof of Work (PoW), the Proof of Elapsed Time (PoET), the Proof of Stake (PoS), Practical Byzantine Fault Tolerance, and Delegated Proof of Stake (DPoS). Each consensus mechanism has different advantages and disadvantages that are related to the node identity management, energy saving, tolerance power of adversary and the needs of applications that are applied on. Finally, the throughput of blockchain systems is defined as the number of transactions that are stored in the ledger per second. Conventional Blockchain systems have limited throughput, like Bitcoin that is limited to seven transactions per second, because of the complexity of the Proof of Work consensus mechanism that it uses [16]. In fig. 2, the way that Blockchain works is presented. Everything starts when a user requests a transaction. The requested transaction is broadcasted to a Peer-to-Peer (P2P) network, where other users-computers, which are referred to as a node, will validate the transaction and the status of the users. Once verified, the transaction 
is combined with other transactions to create a new block of data that will be added to the ledger. In this way, the information that exists in the Blockchain is permanent and unalterable. Furthermore, the sequence of Blockchain/ledger is presented in fig. 3. Blockchain can provide many of its security and privacy features that it obtains, like strong cryptographic techniques, immutability, transparency, and resilience. One of the main features of Blockchain is its distributed nature, which contributes to the resilience of the network [12]. Public Blockchain networks are totally decentralised and distributed. The ledger is stored locally by each node of the network. Furthermore, all the nodes come to a consensus regarding which block will be the next in the chain.

a) Critical Evaluation: Comparing the traditional blockchain architecture to ours, one difference is related to the distributed nature of Blockchain as the ledger will be stored away from the nodes of the network, on Edge Computing nodes. We acknowledge that the distributed nature of Blockchain is relaxed by keeping a single copy of the ledger on Edge Computing nodes. There are two options that can be used and work to address this issue. Our first assumption is that there are more than one Edge Computing nodes, where the ledger can be stored, even though our first assumption is that the Edge Computing service provider is considered to be completely trusted and reliable. This solution has many benefits, as the ledger will be always available and shared among several Edge Computing nodes in the case where one of them is unavailable to serve, and mobile devices can have access to data in any case. Instead of one central Edge Computing server, there are a few more that obtain the same copy of the ledger and interact with the IoT devices of the network in the same way as the first. Basically, there is only one Edge Computing server that has some copies of its own, which are spread around the smart circular city. This solution addresses a second issue, as well, that is related to the distance of devices from the Edge Computing nodes and their ability to access stored data. In this way, mobile devices of the network will be able to operate properly, regardless of their location and how far they are from the Edge Computing servers, as there will be many of them to cover these needs. The second option that could be employed is the use of the Cloud. The assumption here is that the ledger is periodically pushed to a Cloud service provider. The requirements for this option are that the Cloud provider is considered to be reliable, always available and trustworthy. The ledger will be stored at each Edge Computing node and all the IoT devices will be connected to them. In regular time periods, all these Edge Computing nodes will keep the Cloud-stored copy of the ledger updated. This copy is used as a backup. In this way, the safety and availability of the ledger are ensured. Again, one of the fundamental features of Blockchain, which is the decentralisation is relaxed, as the ledger is kept and stored by a central authority, the Cloud in this case, however, we believe that the advantages of our approach are greater than the arising issues as data will always be available to users and the performance of the network will be improved.

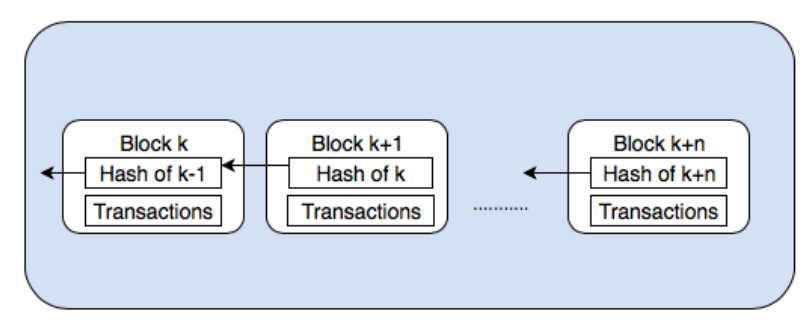

Fig. 3. Blockchain Architecture

\section{B. Edge Computing for Mobile Blockchain}

On the other hand, in [13], authors present a new architecture, in which they use Edge Computing in order to help mobile and IoT devices that run a Blockchain application to compute faster, with consuming less of their resources, the Proof of Work value that miners need. To achieve this, all the nodes of the network, who act as miners, ask from the Edge Computing service provider, which has deployed Edge Computing servers for mining, to compute the Proof of Work value in the account of them. This architecture is presented in fig. 4, where mobile/IoT devices that run a Blockchain application offload the mining process to the Edge Computing units. The mining process that will be conducted by the Edge Computing service provider is secured by solutions like data masking or obfuscation. In exchange for its services, Edge Computing provider asks fees from each miner. If they come in agreement, Edge Computing provider receives the fees that asked from the miner and returns the result of the Proof of Work value computation. The miners receive this value, broadcasting it to the other miners of the network. If all of them consent, the miner adds the new block to the chain and receives the reward for his achievement. This reward is expressed as a utility function. The concept that authors want to approach is related to the reduction of energy and CPU consumption of mobile devices, which run blockchain applications. Devices will consume energy and CPU of them, however, less than in traditional Blockchain networks. Authors use Blockchain and Edge Computing together in order to reduce the energy levels consumption by mobile devices. Even though the results from the tests that the author did, reveal that the efficiency of the architecture is satisfactory, there are some disadvantages regarding the offloaded mining process.

a) Critical Evaluation: As miners may belong to the same Blockchain network, they use the same Edge Computing service provider for computing the Proof of Work value. Thus, they compete with each other, as each one wants to be a miner in order to generate the new block of the chain. In this case, there are some "blind spots" regarding the transparency of the process. Furthermore, the problem regarding the complexity of Proof of Work could be solved by using an alternative consensus mechanism that does not require high levels of CPU and energy consumption. Also, delegating the Proof of Work 


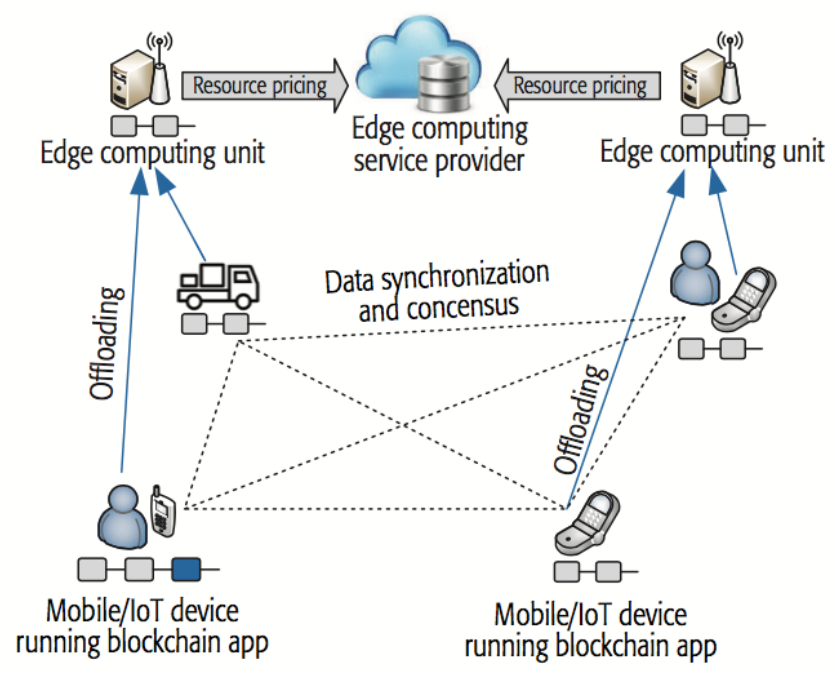

Fig. 4. Edge Computing for Mobile Blockchain [13]

value computation to mobile Edge Computing, malicious users who may be members of the network can have the opportunity to validate their transactions and affect the network and other users. A reason, why, Proof of Work computation is complex, is in order to prevent the performance of this kind of nodes in the network from harming it by validating malicious transactions. We have kept in mind that Blockchain application over IoT networks arises this kind of challenges, however, we believe that the computation of Proof of Work value must be performed by the miners of the network for the reasons that we mentioned above. Blockchain networks operation is based on this process and guarantees that a node is trustworthy, thus, the performance of this process by a different entity, Edge Computing, in this case, could have harmful consequences for the network.

\section{Discussion AND FUtURE WORK}

The recent infrastructural and ICT investment efforts and initiatives to address sustainability challenges in urban environments coincide with the high growth and adoption rate of IoT and associated disruptive technologies such as Blockchain, as well as new networking paradigms such as softwaredefined networks, are constructing the mosaic of emerging technologies for smart cities. Sustainability reminds us that the ultimate goal of a city is not to become "smart" but "circular", where the former term is used as an enabler to achieve the latter. At the same time, the recent research and innovation frenzy towards the adoption of the disruptive and acclaimed technologies such as Blockchain, promises to solve a number of challenges across a variety of smart city use cases and projects. However, a closer look into these new paradigms may reveal that these new technologies are not a panacea; if we consider historical technological milestones and their underpinning economic models and theories, we would notice that many of the proposed applications and solutions are not practical or feasible whereas in some cases they may have adverse effects, contradicting their purpose. For instance, the Jevons Paradox [20] may come into play in the case of autonomous vehicles; the technological advancements of all IT innovations required to fully achieve vehicle autonomy (AI, pioneering $5 \mathrm{G}$ frequencies for communication, big data and machine learning) will result into increased energy demands. As it is safe to assume that autonomous vehicles will be electric - again due to the advancements of research into electric energy efficiency - we will end up having to invest the majority of the vehicle's energy to meet the expensive computations and cooling needs. Naturally, this will have a detrimental effect on the vehicle's range. To add to the mix, Blockchain operations are expensive by nature - or more precisely, expensive by design. Yet Blockchains offer added value by securing the transactions and offering traceability of the high volume of negotiations between the stakeholders, owners, and beneficiaries of the limited resources.

Furthermore, reflecting upon Wriston's Law [18] stating that 'capital', when freed to travel at the speed of light, will go where it is wanted, stay where it is 'well-treated', we could argue that in a data-driven $\mathrm{CE}$ ecosystem, capital is information and as such this will require a suitable architecture to allow the data to travel and identify equilibria and locations where it is best utilised. In a smart city context, we conjecture that a suitable architecture would be a hierarchical Edge Computing. Our future work could focus on Blockchain application on IoT networks, however, based on hierarchical Edge Computing. For instance, the IoT devices that belong to a specific building or service in a smart city can be connected to a sub-Edge Computing that is connected with a bigger one, which has greater range and communicates with all these sub-Edge Computing nodes of this environment. In this way, the performance of these devices could be enhanced more, as the Edge Computing, where they will be connected, will serve fewer devices than a centralised one that covers a city. Furthermore, an experiment will be our next step in order to gather information about the performance evaluation of the prototype.

\section{CONCLUSION}

In this article, we introduce the Edge Computing and how it can contribute to IoT networks that run Blockchain, as the application of it brings some issues along with benefits. The main issue is the limited storage capacity of IoT devices that are located in a smart, circular city and the volume of data that they must keep. To this end, Edge Computing is employed. Devices of this network will be able to communicate with Edge Computing nodes and between each other, and store new blocks of data and transactions not locally, but, on Edge Computing. In this way, it is not necessary to consume their $\mathrm{CPU}$ and they can use these resources in a different way. Thus, we believe that the performance and efficiency of IoT devices will be increased, which is very important in a real-world scenario. Furthermore, by storing data on Edge Computing nodes, the purposes of information circularity in a smart city concept can be fulfilled, as the extraction of data can be 
achieved in order to mitigate the resources consumption and the waste production in order to improve the services of this concept.

\section{ACKNOWLEDGMENT}

This work was partially supported by IDEAL-CITIES; a European Union's Horizon 2020 research and innovation staff exchange programme (RISE) under the Marie SkłodowskaCurie grant agreement No 778229.

\section{REFERENCES}

[1] Horizon 2020, Cyber-Trust, Advanced Cyber-Threat Intelligence, Detection and Mitigation Platform for a Trusted Internet of Things, RIA Research and Innovation Action.

[2] Geissdoerfer, M., Savaget, P., Bocken, N.M. and Hultink, E.J., 2017. The Circular Economy-A new sustainability paradigm?. Journal of cleaner production, 143, pp.757-768

[3] Talari, S., Shafie-Khah, M., Siano, P., Loia, V., Tommasetti, A. and Catalão, J., 2017. A review of smart cities based on the internet of things concept. Energies, 10(4), p.421.

[4] Dorri, A., Kanhere, S.S. and Jurdak, R., 2017, April. Towards an optimized blockchain for IoT. In Proceedings of the second international conference on Internet-of-Things design and implementation (pp. 173178). ACM.

[5] Dorri, A., Steger, M., Kanhere, S.S. and Jurdak, R., 2017. Blockchain: A distributed solution to automotive security and privacy. IEEE Communications Magazine, 55(12), pp.119-125.

[6] Hu, Y.C., Patel, M., Sabella, D., Sprecher, N. and Young, V., 2015. Mobile edge computing-A key technology towards 5G. ETSI white paper, 11(11), pp.1-16.

[7] Zheng, Z., Xie, S., Dai, H., Chen, X. and Wang, H., 2017, June. An overview of blockchain technology: Architecture, consensus, and future trends. In Big Data (BigData Congress), 2017 IEEE International Congress on (pp. 557-564). IEEE.

[8] Damianou, A., 2017. Anonymity on Cryptocurrencies. MSc thesis in Information Security. Royal Holloway, University of London.

[9] Abbas, N., Zhang, Y., Taherkordi, A. and Skeie, T., 2018. Mobile edge computing: A survey. IEEE Internet of Things Journal, 5(1), pp.450-465.

[10] Zheng, Z., Xie, S., Dai, H.N. and Wang, H., 2016. Blockchain challenges and opportunities: A survey. Work Pap.-2016.

[11] Sankar, L.S., Sindhu, M. and Sethumadhavan, M., 2017, January. Survey of consensus protocols on blockchain applications. In 2017 4th International Conference on Advanced Computing and Communication Systems (ICACCS)(pp. 1-5). IEEE.

[12] GSMA, Opportunities and Use Cases for Distributed Ledger Technologies in IoT, 2018

[13] Xiong, Z., Zhang, Y., Niyato, D., Wang, P. and Han, Z., 2018. When mobile blockchain meets edge computing. IEEE Communications Magazine, 56(8), pp.33-39.

[14] Sabella, D., Vaillant, A., Kuure, P., Rauschenbach, U. and Giust, F., 2016. Mobile-edge computing architecture: The role of MEC in the Internet of Things. IEEE Consumer Electronics Magazine, 5(4), pp.8491.

[15] Dr Baru, S., Ratan, N., 2018. Blockchain. The next innovation to make our cities smarter.

[16] Zheng, Z., Xie, S., Dai, H., Chen, X. and Wang, H., 2017, June. An overview of blockchain technology: Architecture, consensus, and future trends. In 2017 IEEE international congress on big data (BigData congress) (pp. 557-564). IEEE.

[17] Ellen MacArthur Foundation, 2016. Intelligent Assets: Unlocking the Circular Economy Potential.

[18] Karlagaard, R., 2005. Ten Laws of the Modern World. Forbes.

[19] Babou, C.S.M., Fall, D., Kashihara, S., Niang, I. and Kadobayashi, Y., 2018, June. Home Edge Computing (HEC): Design of a New Edge Computing Technology for Achieving Ultra-Low Latency. In International Conference on Edge Computing (pp. 3-17). Springer, Cham.

[20] Wikipedia contributors. (2019, March 11). Jevons paradox. In Wikipedia, The Free Encyclopedia. Retrieved 12:56, March 22, 2019.
[21] Blockchain size. [Online]. Available: https://blockchain.info/charts/ blocks- size 